\title{
Preliminary Application of the Theory of Electroacupuncture Along the Meridian to the Treatment of Cerebral Hemorrhage Hemiparalysis
}

\author{
Xuejian Wang, Zhifeng Wang, Yang Chen, Ming Qian \\ Department of Neurosurgery, The Second Hospital Affiliated to Nantong University, Nantong University, Nantong, PR China
}

Email address:

6841441@163.com (Xuejian Wang)

\section{To cite this article:}

Xuejian Wang, Zhifeng Wang, Yang Chen, Ming Qian. Preliminary Application of the Theory of Electroacupuncture Along the Meridian to the Treatment of Cerebral Hemorrhage Hemiparalysis. American Journal of Psychiatry and Neuroscience. Vol. 5, No. 5, 2017 , pp. 53-55. doi: 10.11648/j.ajpn.20170505.11

Received: June 5, 2017; Accepted: August 10, 2017; Published: September 7, 2017

\begin{abstract}
Hemiparalysis is a common symptom of cerebral hemorrhage. Chinese traditional acupuncture and electroacupuncture are two major treatments for the disease. However, they often fail to achieve satisfactory outcome. It reports here successful treatment of three patients with hemiparalysis using electroacupuncture along the meridian therapy along with prescription medicines to restore the muscle strength. The three patients had a history of hemiparalysis and were diagnosed hemiparalysis due to cerebral hemorrhage based on CT or MRI examinations. Acupuncture was applied to restore the muscle strength with routine treatment and prescription medicines. The patients were treated 20 times with electroacupuncture along the meridian. After 1 months of the treatment, case 1 and case 2 regained the muscle strength, and case 3 was remarkably recovered. It is likely that combining Chinese tradition acupuncture with electroacupuncture is more effective to treat hemiparalysis, although more studies are needed to validate the approach.
\end{abstract}

Keywords: Electroacupuncture, Acupuncture, Meridian, Acupoint Selection, Hemiparalysis, Acupuncture along the Meridian, Electroacupuncture Along the Meridian

\section{Introduction}

Acupuncture along the Meridian is a common and effective acupuncture method in traditional Chinese acupuncture. It is a proven approach for treating hemiparalysis of cerebral hemorrhage $[1,2]$. Electroacupuncture (EA) is introduced to China from abroad China. Due to its excellent sensation and treatment effect, it has been perfectly combined with the traditional acupuncture. However, the EA is applied at fixed points of stimulation with the same frequency and strength, its stimulation may not able to reach the lesion sites distal to the acupoints, because the sensation is limited to the area near the acupoint. This, to some extent, limits its efficacy. On other hand, the sensation obtained through the traditional acupuncture can circulate along the meridian [1, 3, 4, 5]. We have proposed a theory of 'EA along the meridian', which suggests to adjust the EA probe move gradually along the meridian from distal acupoint to proximal acupoint to transfer the sensation from distal acupoint to proximal acupoint. This is a valuable combination of traditional acupuncture theory and modern EA technology. We report here the successful treatment of 3 patients with hemiparalysis of cerebral hemorrhage using the technique.

\section{Case Presentation}

\subsection{Ethics Approval and Consent to Participate}

This research has been approved by the ethics committee of the Second Hospital affiliated to Nantong University (2016002). Informed consent has been obtained and this investigation has been conducted according to the principles expressed in the Declaration of Helsinki. And the authors have obtained written informed consent of all the patients.

\subsection{History and Examination}

Case one: Patient Huang was a male clerk aged 46. He was admitted due to sudden headache and left hemiplegia for 3 
hours. The patient had a history of hypertension. Examination showed that he was conscious and active with muscle strength II in the left upper limb and III in the lower limb. Pathological and CT examinations showed that he was PAP positive with hemorrhage in the right basal ganglia.

Case two: Patient Yang was a 52 year old business private owner. She was admitted due to sudden fatigue of right limb for 2 hours and had a history of hypertension and coronary heart disease. She was conscious and active with muscle strength IV in the right upper limb and IV in the lower limb. Pathological and CT examinations showed that she was PAP positive with hemorrhage in the left basal ganglia.

Case three: Patient Cai was a 59 year old farmer and admitted due to sudden unconscious for 3 hours. He had no prior physical examination and denied any history of disease. He was delirious and not active to following the doctor's instructions. CT examinations showed that he had hemorrhage in the left basal ganglia. He was given emergency surgery and was right limb hemiparalyzed with muscle strength II in both the upper and lower limbs after the surgery.

\subsection{Treatment}

The patients were treated with EA long the meridian technique. For the upper limbs, the acupoints were Hegu, Shousanli, Quchi, Shouwuli and Jianyu, for the lower extremities, the acupoints were Xiajuxu, Yanglingquan, Zusanli, Sanyinjiao, Yongquan and Yongquan. The EA was applied gradually from distal to proximal acupoints at a frequency of 500 times/min for 2 to 3 minutes at each point. 6 to 8 minutes later, the patients were stimulated with new rounds of the same EA for a total 40 minutes. After rested for 5 to 10 minutes, the EA treatment is terminated. The EA procedure was given once a day for 10 to 15 days with a resting interval of one week for next round of treatment.

After two rounds of treatment, the patients were assessed for muscle strength. From the evaluation of the efficacy of the treatment after the course of treatment, muscle strength recovery in the case one and two were up to grade $\mathrm{V}$, cases three up to IV level, and the treatment effect is satisfactory.

\section{Results and Discussion}

Acupuncture is a traditional Chinese medicine technique. It is reliable, minimally invasive with complex mechanisms. The mechanisms are mainly based on the traditional theory of meridian, which is the core of the basic theory of traditional Chinese medicine. One of the main function of the meridian is to participate in the connection of the organs and regulation of body function and reaction $[4,6]$. Meridian is located with the bowel, connected to the limbs and join the Yin and Yang [7, 8].

Previous studies indicated that the temperature, oxygen partial pressure and energy metabolism in the deep tissues along the meridian lines are higher than those along the corresponding non-meridian lines $[9,10]$. $\mathrm{Xu}$ et al found that the blood flow was increased in the range lines of meridian when acupuncture was applied $[9,10]$, which was similarly reported by others $[11,12]$. These results suggest that EA may increase the blood microcirculation in the range lines and the effect of EA may partially result from the meridian theory of traditional medicine. Zhang et al investigated the high temperature lines along the meridian after applying acupuncture, EA, moxibustion, fire-acupuncture and catgut embedded in the acupoint using infrared thermography in rabbits and found that the temperature incensement was the highest $\left(0.7\right.$ to $\left.1.0^{\circ} \mathrm{C}\right)$ after moxibustion, followed by EA $\left(0.5\right.$ to $\left.0.8^{\circ} \mathrm{C}\right)$, and lasted 5 to $30 \mathrm{~min}$. They concluded that the high-temperature line along the meridian is induced most effectively by moxibustion and EA, suggesting that EA may increase the therapeutic effect of traditional acupuncture. EA therapy is a modern method, which generates acupuncture and electric stimulations with high strength. The rapid development of acupuncture both within and outside China over the last few decades has itself led to great innovations in practice. When the traditional theory of acupuncture is assisted with modern medical measures, such as EA, would reduce the accuracy requirement in acupuncture for better efficacy. However, previously, when EA was applied, it was only considered to provide consistent electrical stimulation and has not taken consideration to apply along the meridian as does in the traditional medicine nor performed as hand acupuncture, where the needles are moved up and down and turned around. As such, the stimulation from EA is unable to reach the lesion site in the distal acupoints and is only effective round the acupoints. Therefore, the efficacy of sensation therapy is limited [14, 15].

Based on the above theory, we proposed a theory of 'EA along the meridian'. It requires to select the acupoint along the meridian and defines the direction and depth of needle before EA, and then EA needle is connected to the instrument and applied to the acupoint sequentially along the meridian to treat. This makes a better use of traditional meridian theory and modern acupuncture technology, and is a step forward technically. We have demonstrated here that is clinically effective, although more clinical works are needed to validate it and to differentiate it from existing technology.

\section{Conclusion}

This is only a few cases and electroacupuncture follow by meridian was used in combination with other therapies. This report suggests that there may be a more role to combined with Chinese tradition acupuncture and electroacupuncture to get a good effective, and a welcome addition to our information if more case studies about the possibility of acupuncture use in these circumstances are done.

\section{Acknowledgements}

This research supported by: Young Medical Project of 
Nantong City Health Bureau (WQ2014016); Traditional Chinese medicine science and technology project in Jiangsu province (YB2015113); The science and technology program of Nantong City (MS12015016).

\section{References}

[1] Lewith GT, White PJ, Pariente J. Investigating acupuncture using brain imaging techniques: the current state of play. Evid Based Complement Alternat Med 2005; 2: 315-9.

[2] Shi YuanSheua, Chun HsuYaoa, Yuan TsungFua, Wen LingWanga Acupuncture as complementary therapy for hypoxic encephalopathy: A case study. Complementary Therapies in Medicine 2010; 18, 265-268.

[3] Lin Lijiao, Xu Jinsen, Zhu Xiaoxiang, Zheng Shuxia. Development on research of acupuncture effects on microcirculation $[\mathrm{J}]$. Chinese Acupuncture \& Moxibustion, 2015, 35(2): 203-208.

[4] Liu T. Role of acupuncturists in acupuncture treatment. Evid Based Complement Alternat Med 2007; 4: 3-6.

[5] Kaptchuk TJ: Acupuncture: theory, efficacy, and practice. Ann Intern Med 2002, 136: 374-383.

[6] Tan S, Tillisch K, Mayer E. Functional somatic syndromes: emerging biomedical models and traditional Chinese medicine. Evid Based Complement Alternat Med 2004; 1: 35-40.

[7] Liu G, Akira H. Basic principle of TCM. In: Liu G, Akira H, editors. Fundamentals of acupuncture and moxibustion. Tianjin: Tianjin Science and Technology Translation and Publishing Corporation; 1994.
[8] Jiao SF. Scalp acupuncture and clinical cases. 3rd ed. Beijing: Foreign Languages Press; 2005.

[9] Gao Lingyun, Hu Xianglong, Xu Xiaoyang, Wu Baohua, Chen Ling. Measurement of the Temperature in Deep Tissues along the Governor Vessel [J]. Acupuncture Research, 2006, 31(3): 159-162.

[10] Chen Ming, Hu Xianglong, Wu Zuxing. Effect of Acupuncture on Partial Oxygen Pressure of Deep Tissue Along the Governor Vessel in 31 Normal Volunteer Subjects [J]. Acupuncture Research, 2008, 33(6): 402-405.

[11] Wang Peiqing, $\mathrm{Hu}$ Xianglong, $\mathrm{Xu}$ Jingseng. Infrared Image-Displaying of the Fourteen Meridian Courses [J]. Acupuncture Research, 2002, 27(4): 260-261.

[12] Yang Hongqin, Xie Shusen, Hu Xianglong. Appearance of human meridian-like structure and acupoints and its time correlation by imfrared thermal imaging $[\mathrm{J}]$. American Journal of Chinese Medicine, 2007, 35(2): 231-240.

[13] Zhang Dong, Fu Weixing, Wang Shuyou, Ma Huiming, Wang Yuanchao. Comparison of Phenomenan of Highthermal Lines along Channels Induced by Different Acupuncture-Moxibustion Methods [J]. Chinese Acupuncture \& Moxibustion, 2006, 6: 349-353.

[14] Bao Yuanshi. Developlnent and usage of the Along-Meridian Treatment Instrument [J]. Chinese Acupuncture \& Moxibustion, 2009, 29(4): 337.

[15] Wang YJ. Micro-acupuncture in practice. Missouri: Churchill Livingstone; 2008. 